zug oder zur Fusion mit Union oder SPD bewegen würde. ${ }^{11}$ Auch unter Berücksichtigung psychologischer Wahlsystemeffekte erscheint es somit zweifelhaft, ob die gemäßigte Mehrheitswahl Einparteiregierungen herbeiführen kann. ${ }^{12}$

Als Fazit bleibt festzuhalten, dass das proportional ergänzte Mehrheitswahlsystem kleine Parteien im Bundestag empfindlich zugunsten großer Parteien schwächt. Zugleich ist es nicht in der Lage, in der Bundesrepublik Deutschland unter den gegebenen Bedingungen eine Parlamentsmehrheit für eine Partei herbeizuführen - und das dürfte es unter Strohmeiers Annahmen ${ }^{13}$ auch künftig nicht leisten können. Selbst wenn man die Prämissen ihres Verfechters zugrunde legt, vermag die gemäßigte Mehrheitswahl somit nicht zu überzeugen. Daher sind ihre Chancen, die personalisierte Verhältniswahl in absehbarer Zeit als Bundestagswahlsystem abzulösen, als gering einzuschätzen.

11 Dabei ist unter anderem zu bedenken, dass mit der Einführung der gemäßigten Mehrheitswahl auf Bundesebene dieses System nicht automatisch auch auf Landesebene eingeführt würde.

12 Analog ändert die Berücksichtigung psychologischer Effekte das Ergebnis zu den kleinen Parteien nicht wesentlich.

13 Vgl. Gerd Strohmeier, a.a.O., S. 581.

\title{
Mit dem Internet in die parlamentarische Provinzialität
}

\author{
Erich Röper
}

Als Voraussetzung demokratischer Willensbildung fordern das Grundgesetz und die Landesverfassungen die Öffentlichkeit der Parlamentssitzungen. Es gilt folgerichtig für alle parlamentarischen Vorgänge, jedenfalls grundsätzlich, da etwa die Sitzungen der Ausschüsse meist nichtöffentlich sind. Auch die den Plenardebatten zugrunde liegenden Drucksachen müssen allgemein zugänglich sein, den Abgeordneten aller Parlamente, ihren Fraktionen und Mitarbeitern sowie Interessierten. Obwohl sie in allen Parlamenten, Fraktionen und vielen Bibliotheken vorlagen, erreichten sie in Papierform nur einen beschränkten Personenkreis. Daher gilt das Internet als Königsweg.

\section{Parlamentarische Dokumente in Papierform: Chance auf umfassende politische Kenntnis}

Die Möglichkeit für Abgeordnete und Fraktionen, sich einen Überblick über das parlamentarische und politische Geschehen in Bund und Ländern zu verschaffen, wurde seit einiger Zeit eingeschränkt: Seit dem Jahr 2002 stellten der Bundestag und die Landtage den Drucksachenaustausch nach und nach ein, seit 2005 auch ihren Versand an die meisten Parlamentsbibliotheken. Der Austausch wurde auf das Internet konzentriert; Papierexemplare erhalten nur noch der Bundestag, der Bundesrat und der Landtag von Niedersachsen. 
Viele Jahre erhielten auch die Fraktionen, sofern wie vom Autor gewünscht ${ }^{1}$, die Drucksachen und Plenarprotokolle der anderen Parlamente. Die Landtage der östlichen Bundesländer vollzogen den Drucksachenaustausch von vornherein nicht mit. Nach und nach stellten jeweils mit dem Ende ihrer Wahlperiode auch die Landtage im Westen, zuletzt der Bundestag - seine Dokumente wurden auch an Universitäten versandt - den Informationsdienst für die Fraktionen ein, vor allem um die Versand- und Satzkosten einzusparen, die je nach Ausgestaltung verschieden sind. Eine Rolle spielten auch räumliche und personelle Kapazitätsgesichtspunkte und eine vergleichsweise geringe Nachfrage.

Der Internetauftritt www.parlamentsspiegel.de dient als Ersatz für die noch in den siebziger Jahren versandten Papierdokumentationen und späteren Mikrofiches. Die Jahreskosten von rund $€ 350.000$ wurden auf die Landtage nach dem Königsteiner Schlüssel umgelegt. Die Kosten konnten durch eine Vereinheitlichung der Dokumentenerfassung auf rund $€ 100.000$ gesenkt werden. Dennoch stiegen Baden-Württemberg und Hessen im Januar 2004 aus dem Parlamentsspiegel aus; ihre Dokumente finden sich seither nur noch in den Datenbanken dieser Landtage. Während die übrigen Landesparlamente den Parlamentsspiegel als Datenbank beibehalten wollten, plädierten Stuttgart und Wiesbaden für ein bloßes Internetportal durch die Verbindung der Links von den Homepages der einzelnen Landtage. Es gibt jedoch ernsthafte Bemühungen, diese zwei Länder wieder ins gemeinsame Boot zu holen.

Viele Jahrzehnte war es gute Übung, die Arbeit des Bundestags und der Landtage anhand der Dokumente, der Anfragen aller Art und Anträge zu verfolgen. Die Fraktionen bekamen die Tagesordnungen, Drucksachen und Protokolle der anderen Volksvertretungen in Papierform. Die Abgeordneten und Mitarbeiter konnten sie auswerten. Mit konsequenter Durchsicht der Drucksachen erkannten sie genauer als aus den Plenardebatten die Ziele und Inhalte des politischen Handelns. Sie lernten von den Initiativen oder Rückschlägen der Kollegen und erkannten früh den Versuch politischer Gegner, mit gleichgerichteten Initiativen politische Themen zu besetzen. Sie entnahmen den Antworten der Regierungen - manchmal mit der Qualität von Kurzgutachten - Wissenswertes für die eigene Arbeit und den Stand der Politik in der Republik. Wer je wie etwa der Autor die Bundestagsdrucksachen mit den Antworten auf die schriftlichen Anfragen durchgesehen hat, weiß um den Schatz des dort niedergelegten politischen, wirtschaftlichen und juristischen Sachwissens für die politische (und wissenschaftliche) Arbeit $^{2}$.

\section{Internet-Monopol im parlamentarischen Betrieb: Orwell'sches Gedankenloch}

All das ist Geschichte. Das Internet soll es richten. Alles könne im Netz recherchiert werden. Das ist grundsätzlich ohne nennenswerte Kosten möglich. Am Bildschirm können Drucksachennummer für Drucksachennummer durchgesehen, Vorgänge in den Dokumenten ver-

1 Kurz nach dem Beginn der über 25-jährigen hauptamtlichen Tätigkeit für die CDU-Fraktion der Bremischen Bürgerschaft, davon über 20 Jahre als Geschäftsführer, erhielt der Autor regelmäßig alle Protokolle des Bundestags, des Bundesrats und der Landtage sowie ihre Drucksachen und wertete sie für die Arbeit der Fraktion und ihrer Mitglieder aus.

2 Zur Inhaltsanalyse von Parlamentsdrucksachen und ihrer empirischen Bedeutung Jens Kalke, Bedeutungsverlust der Landtage? Ein empirischer Test anhand der Drogenpolitik, in: ZParl, 32. Jg. (2001), H. 2, S. 309 ff., S. 314 f. 
gangener Wahlperioden erforscht werden, ohne in eine Landtagsbibliothek zu fahren. In der Praxis verringert die Beschränkung auf diese Technik jedoch den Informationsfluss. Für die Fraktionen und Abgeordneten entfällt die umfassende Kenntnis der politischen Arbeit in den anderen Parlamenten. Sie beschränkt sich nunmehr auf die eigene Volksvertretung sowie Medienberichte und fördert das Denken und Handeln mit Scheuklappen.

Das Internet ist kein Ersatz. Auch im kaum denkbaren Fall, dass Fraktionen die sich in den Kleinen und Großen Anfragen oder schriftlichen Auskunftsersuchen, den Anträgen und Gesetzesvorschlägen manifestierende Politik ${ }^{3}$ am Bildschirm verfolgen, hätten sie hunderte von Seiten auszudrucken, um ihre Inhalte vergleichen zu können. Nur eine anscheinend ziellose Recherche erlaubt den Einblick in das scheinbar strukturlose politische Geschehen. Im Internet ist Vorgängen nur mit vorgegebenem Ziel nachzugehen. Etwas finden werden nur die, die einem besonderen Thema auf der Spur sind. Auch dann häufen sich die Probleme. Einen einheitlichen Internetauftritt der Parlamente gibt es nicht. Die Homepages der Landtage sind verschieden aufgebaut und erlauben kaum die Suche nach den gleichen Begriffen.

Für Querschnittsinformationen über alle Länder bleibt nur die Einzelrecherche über die jeweiligen Seiten der Landesparlamente und eingeschränkt über den Parlamentsspiegel. Das gleiche gilt für die Links zu den Parlamenten untereinander. Auch hier sind die vorherige Kenntnis der Wahlperiode und Drucksache oder / und die genaue Bezeichnung des gesuchten Dokuments nötig. Insbesondere „Zufallsfunde“ sind nahezu ausgeschlossen. Es gibt nur begrenzt eine einheitliche Verschlagwortung, und kaum etwas ist mit ungefähren Begriffen zu ermitteln. Jedes Parlament werkelt vor sich hin und übernimmt ins Sach- und Sprechregister die von der Regierung, den Fraktionen oder Abgeordneten jeweils genannten Betreffpunkte, soweit diese die Parlaments-Dokumentatoren nicht gegebenenfalls mit dem Ziel einheitlicher Begriffe ändern, ergänzen oder angleichen.

Es gibt Dokumentatorentreffen und Redaktionskonferenzen für den Parlamentsspiegel zur Standardisierung der Leitbegriffe und der Betreffpunkte der jeweiligen Drucksachen. Die Internetseiten der Landtage bieten einen Anwendungsthesaurus für die Schlagworte. Der von Nordrhein-Westfalen für die Landtage betriebene Parlamentsthesaurus ist nichtöffentlich und dient der Arbeit der Dokumentatoren. Nur potentiell sinkt mit der Beschränkung auf das Internet die Exklusivität von Informationen und steigt die Transparenz. Viele Vorgänge verschwinden im Orwellschen Gedankenloch.

\section{3. „Zufallsfunde“ politischer Vorgänge und Skandale ausgeschlossen}

Wo sind etwa die Funktionszulagen der Fraktionshierarchen in den überall anders getitelten Rechenschaftsberichten der Fraktionen zu finden? ${ }^{4}$ Nur die werden sie aufdecken, die schon vorher die Fundstelle wissen.

Ärger noch ist es bestellt um wichtige politische Sachverhalte, deren parlamentarische Behandlung nur zufällig aufgedeckt wird. Wer käme auf die Idee, dass in Bayern die Witwe

3 Ein berühmtes Beispiel ist der SPD-Bundestagsabgeordnete, Landesvorsitzende und Bundesschatzmeister Wilhelm Dröscher, der "gute Mensch von Kirn“, der nie im Bundestagsplenum sprach, sondern mithilfe immer neuer Anfragen regionale und nationale Politik betrieb.

4 Dazu Erich Röper, Umwegfinanzierung der Fraktionshierarchien. Eine Replik auf Gerald Kretschmer in Heft 4/2000 der ZParl, in: ZParl, 34. Jg. (2003), H. 2, S. 419 ff. 
von Adolf Hitlers Volksgerichtshofpräsidenten Roland Freisler als Schadensausgleich nach $\$$ 40a BVG/DVO zu $\$ 30$ Abs. 3, 4 BVG (unselbständige Tätigkeiten in der privaten Wirtschaft bei abgeschlossenem Hochschulstudium) eine erhöhte Hinterbliebenenrente erhält ${ }^{5}$ ebenso wie die eines am 7. Juni 1951 hingerichteten SD-Einsatzkommandoführers, gelangt nicht die Landtagsdrucksache auf den Tisch? Wer würde wissen, wie viele SS- und NSDAP-Mitglieder nach 1945 Hamburgs Polizei übernommen hat ${ }^{7}$ oder wie großzügig dieser Staat mit Versorgungsempfängern umgeht, die 1933/45 im öffentlichen Dienst gegen die Grundsätze der Menschlichkeit oder Rechtsstaatlichkeit verstießen, aber für die Zeit, in der sie vor Ablauf des 8. Mai 1945 wegen der Beschäftigung im öffentlichen Dienst versicherungsfrei waren, als in der gesetzlichen Rentenversicherung nachversichert gelten bis zur Höhe der damaligen Beitragsbemessungsgrenze, hätte nicht der Finanzminister von Rheinland-Pfalz es offenbart ${ }^{8}$ ?

Wer surft regelmäßig durch die parlamentarischen Internetdokumente auf der Suche nach Informationen über die positive Bewertung der DDR-Lehrer in Nordrhein-Westfalen vor $1990^{9}$, derweil sie heute abgelehnt werden, oder Bayerns Versuche, die Wirtschaftsbeziehungen zur damaligen DDR und nun zu den östlichen Bundesländern einzuschränken? ${ }^{10}$ Wer erführe, dass die Bundesregierung das Urteil des Bundesarbeitsgerichts ${ }^{11}$, nach dem der ordre public in Art. 30 EGBGB nicht verletzt werde, wenn allgemeinverbindliche Tarifverträge auf jugoslawische Gastarbeiter nicht angewendet werden, auf die hier arbeitenden DDR-Deutschen ausdehnte und sie so als Gastarbeiter wertete? Wer weiß vorher, dass der Berliner Senat politisch Verfolgte aus der DDR nicht bevorzugt einstellen wollte ${ }^{12}$, oder von der erfolgreichen SSW-Klage beim Bundesverfassungsgericht für die Befreiung von der FünfProzent-Sperrklausel, hätte es nicht in der einschlägigen Parlamentsdrucksache gestanden ${ }^{13}$ ? Wer könnte wissen von der großen und rasch zunehmenden Zahl der Bund-Länder- und

5 Vgl. Antwort des Bayerischen Staatsministeriums für Arbeit und Sozialordnung, LT-Drs. 10/6016 vom 15./18. Februar 1985.

6 Vgl. Antwort des Bayerischen Staatsministeriums für Arbeit und Sozialordnung, LT-Drs. 10/7679 vom 26./31. Juli 1985.

7 Vgl. Antwort des Senats auf eine schriftliche Kleine Anfrage vom 26. Mai 1987, Bgsch-Drs. 12/698.

8 Vgl. Antwort des Finanzministers Rheinland-Pfalz auf eine Kleine Anfrage, LT-Drs. 10/1448 vom 7. Mai 1985. Siehe auch Antwort des Parlamentarischen Staatssekretärs Horst Günther zu Frage 2, Nr. 3 in der Fragestunde, BT-Prot. 12/154 vom 28. April 1993, S. 13165.

9 Vgl. Antworten der Landesregierung Nordrhein-Westfalen auf Kleine Anfragen, LT-Drsn. 10/1358 vom 18. September 1986 und 10/2899 vom 18. Februar 1988.

$10 \mathrm{Vgl}$. Antwort des Bayerischen Staatsministeriums für Wirtschaft und Verkehr vom 13./15. Januar 1986 auf eine Anfrage zur Beschränkung der Wirtschaftsbeziehungen zur DDR in der Industrie, LT-Drs. 10/8955; Beschluß des Bayerischen Landtags zu Schlachtvieheinfuhren, LT-Drs. 10/11251 vom 22. Juli 1986; zum Rindfleischmarkt Antwort des Staatsministeriums für Ernährung, Landwirtschaft und Forsten vom 5./19. März 1986, LT-Drs. 10/9599; Beschluß des Bayerischen Landtags zur Beendigung der Bezuschussung der Textil-Fachmesse „Contracting Leipzig” aus Bundesmitteln, LT-Drs. 13/11891 vom 8. Juli 1998.

11 Vgl. Antwort des Parlamentarischen Staatssekretärs Wolfgang Vogt vom 7. Juli 1987 auf eine Schriftliche Anfrage, BT-Drs. 11/608. S. 39 ff., S. 41, zu BAG, NJW 1977, S. 2039 f.

12 Vgl. Mitteilung - zur Kenntnisnahme - des Berliner Senats vom 28. Juli 1998 über bevorzugte Einstellung in der DDR politisch Verfolgter, Abghaus-Drs. 13/2960.

13 Dazu Erich Röper, Up ewig ungedelt? - Zum Wahlrecht der dänischen Minderheit in SchleswigHolstein, in: NordÖR 2003, S. 391 ff., aufgrund Antwort der Parlamentarischen Staatssekretärin Ute Vogt vom 7. November 2002 auf eine schriftliche Anfrage, BT-Drs. 15/102, S. 3. 
Länder-Länder-Ausschüsse ${ }^{14}$, die sich jeder parlamentarischen Kontrolle entziehen, oder von der Beteiligung der Landesparlamente bei Verträgen, Vereinbarungen beziehungsweise Abkommen mit anderen Bundesländern, dem Bund und sonstigen Vertragspartnern ${ }^{15}$ ? Und ist es nicht wichtig zu verfolgen, wie zentral geführt die DVU-Landtagsfraktionen sich in ihren Initiativen darstellen oder welch Geistes Kind die NPD-Anträge sind? Die Themen vor allem aus den Kleinen und Schriftlichen Anfragen sind Legion, die im Plenum nicht debattiert und nicht in die Plenarprotokolle aufgenommen werden ${ }^{16}$.

\section{Vor allem die Landtage drohen in Provinzialität und Nabelschau zu versinken}

Die Angaben über solche „Zufallsfunde“ sind aus vergangenen Legislaturperioden. Mangels Drucksachenaustausch war nichts Neueres zu finden. Zweifelsohne ist nicht jede Durchsicht parlamentarischer Dokumente eine Sternstunde. Doch war sie deutlich mehr als die Suche nach der Stecknadel im Heuhaufen. Auch konnten die von den Mitarbeitern mit dokumentarischem Detailwissen ausgestatteten Abgeordneten daheim, bei Sprecherkonferenzen oder ähnlichen Treffen sachkundig über die politische Situation in den anderen Parlamenten mitberaten. Heute sind sie auf Informationen der Gesprächspartner oder dürre Medienberichte angewiesen.

Keinen Ersatz bilden die Berichte und Dokumentationen in den Informationszeitschriften des Bundestags (Bundestag intern), der Zeitung Das Parlament ${ }^{17}$ und die in langen Abständen erscheinenden Schriften einiger Landtage, wie die von Nordrhein-Westfalen, Sachsen, Sachsen-Anhalt, Schleswig-Holstein und Thüringen, oder die Dokumentationen wie in Baden-Württemberg. Sie offenbaren die eine oder andere meist aktuelle Initiative, enthalten gelegentlich Hintergrundberichte, erlauben aber keinen Blick hinter die Kulissen. Nur dieser gibt der Politik aber die nötige Transparenz. Nicht gezielte, von Parlaments- und Fraktionspressestellen ausgewählte Berichte ermöglichen die Beurteilung politischer Arbeit im Positiven wie im Negativen: Wie die Fernsehrealität schiebt sich die so geschaffene Realität vor die Wirklichkeit. Letztere offenzulegen ist auch eine Aufgabe der Wissenschaftler, zumal jener, die Parlamentsrecht und / oder -politik bearbeiten und lehren; ihnen obliegt daher die systematische Auswertung aller Parlamentsdrucksachen und Protokolle, vor allem auch die Suche nach „Zufallsfunden“. Manchmal lassen ihre Aussagen die nötige Detailkenntnis und Informationsbreite insbesondere über die Arbeit der Landtage vermissen.

14 Vgl. zur Entwicklung Antwort der Bayerischen Staatskanzlei auf eine Schriftliche Anfrage zu Bund- und Länderkommissionen, die 356 nennt, LT-Drs.10/8885 vom 30. Dezember 1985 / 7. Januar 1986, auf heute fast 1000, siehe Politikverflechtung in Deutschland, Einbindung des Landes Nordrhein-Westfalen in Abstimmungsprozesse der Länder, Antwort der Landesregierung auf eine Kleine Anfrage, LT-Drs. 13/4187 vom 25. Juli 2003; siehe auch Antwort der Niedersächsischen Staatskanzlei vom 30. Juli 1998 auf eine Kleine Anfrage zu Länderkonferenzen und Bund/ Länder-Kommissionen, LT-Drs. 14/176.

15 Vgl. Antwort des Chefs der Staatskanzlei Rheinland-Pfalz auf eine Kleine Anfrage, LT-Drs. 14/2165 vom 2. Mai 2003.

16 Bis 1975 wurden sie zum Beispiel in den Plenarprotokollen der Bremischen Bürgerschaft aufgeführt.

17 Die Zeitung verzichtet bei der Dokumentation von Plenardebatten und den Berichten über die Beratungen der Ausschüsse auf die Angabe oder den Abdruck der ihnen zugrunde liegenden Drucksachen. 
Die Bedeutung des Internetauftritts der Parlamente soll nicht kleingeredet werden. Dieser erlaubt auch interaktives Handeln etwa mithilfe der vom Bundestag eingeführten elektronischen Petition. Zudem sollten die Bemühungen fortgesetzt werden, die Suchfunktionen, vor allem die Verschlagwortung zu verbessern, soweit der Föderalismus einheitliche Begriffe erlaubt. So gibt es in Bayern keine Großen Anfragen, sondern traditionell Interpellationen, in Hamburg keine Kleinen, sondern nur Schriftliche Anfragen, nur in Baden-Württemberg und Bremen ausführliche Petitionsberichte, sonst existieren nur Sammelvorlagen ${ }^{18}$.

Beide Verfahren müssen beibehalten werden: die Papierform und die Verbreitung der Dokumente im Internet. Natürlich erhöht die Möglichkeit der schnellen Suche nach einer Drucksache oder einem Plenarprotokoll, deren Betreff, Drucksachennummer oder Datum bekannt sind, die Transparenz. Natürlich kann eine einheitliche Verschlagwortung helfen, in einem Parlamentsspiegel wirklich aller Parlamente auf einen Schlag einen für alle geltenden Begriff zu finden. „Zufallsfunde“ aber werden erst ermöglicht, wenn man Papier wälzt.

Keine Fraktion kann zu systematischer Auswertung des politisch-parlamentarischen Geschehens im Bundestag und in den Landtagen gezwungen werden. Zu oft verlassen sie sich auf die Informationen in Geschäftsführer- und Sprecherkonferenzen oder den Versand der Tagesordnungen. Doch ist das Geheimnis guter parlamentarischer Arbeit - und ihrer wissenschaftlichen Auswertung - die Chance zu regelmäßiger, zunächst ungezielter, auf kein vorgegebenes Thema festgelegter Information. Das ist vorbei. Insbesondere die Landtage drohen mit der Beschränkung auf das Internet noch mehr in Provinzialität und Nabelschau zu versinken.

18 Dazu Erich Röper, Fast überall unzureichende Berichte der Petitionsausschüsse, in: ZParl, 33. Jg. (2002), H. 2, S. 239 ff.; ders., Unzureichende Berichte auch des Hamburger Eingabenauschusses. Eine Antwort auf Jürgen Klimke in Heft 4/2002 der ZParl, in: ZParl, 34. Jg. (2003), H. 2, S. 427 ff. 